\title{
miRNA dysregulation and the risk of metastasis and invasion in papillary thyroid cancer: a systematic review and meta-analysis
}

\author{
Tiantian Wang ${ }^{1}$, Hao Xu ${ }^{1}$, Ming $Q^{i}{ }^{1}$, Sheng Yan ${ }^{2}$ and Xingsong Tian ${ }^{1}$ \\ ${ }^{1}$ Department of Breast and Thyroid Surgery, Shandong Provincial Hospital Affiliated to Shandong University, Jinan, Shandong, \\ China \\ 2 Department of Neurosurgery, The First Hospital Affiliated to Sun Yat-sen University, Guangzhou, Guangdong, China \\ Correspondence to: Sheng Yan, email: yanshengpaper@163.com
}

Xingsong Tian, email: tianxingsongpaper@163.com

Keywords: miRNA, invasive thyroid cancer, metastasis, meta-analysis

Received: November 18, 2016 Accepted: March 13, $2017 \quad$ Published: March 29, 2017

Copyright: Wang et al. This is an open-access article distributed under the terms of the Creative Commons Attribution License 3.0 (CC BY 3.0), which permits unrestricted use, distribution, and reproduction in any medium, provided the original author and source are credited.

\section{ABSTRACT}

Thyroid cancer (TC) is the most common endocrine malignancy, with an incidence continuing to grow every year. Although thyroid cancer as a whole is generally indolent and relatively easy to treat, some subtypes carry a higher rate of metastasis and cancer-related mortality. A growing number of studies have focused on the dysregulation of miRNAs in TC. However, differences in methods make comparison of gene profiling data difficult. A meta-analysis of published studies comparing miRNA expression data of invasive thyroid carcinoma with paired non-invasive tumors or normal tissues was performed by searching the literature for "invasion", "thyroid cancer", and "miRNA". This revealed 29 dysregulated miRNAs associated with TC in 16 articles; the presence of invasion was confirmed in each respective article by laboratory research or patient follow-up. Among these miRNAs, miRNA146b, miRNA-221, and miRNA-222 were analyzed further due to their higher frequencies across multiple studies. Of these studies, 6 were included in the meta-analysis, as they compared invasive PTC with paired normal tissues or non-invasive PTC.

\section{INTRODUCTION}

Thyroid carcinoma (TC) is the most common endocrine malignancy, the incidence was increasing year by year. Papillary thyroid cancer(PTC) takes up more than $80 \%$ of TC. The overall survival rate of patients with papillary thyroid carcinoma is high [1], however, increased cancer recurrence and cancer-related mortality are noted in a portion of patients with papillary thyroid carcinoma [2]. Distinguishing these tumors from classic tumors has therefore become a hot topic of research in recent years.

MicroRNAs (miRNAs) are a recently identified class of small, endogenous, non-coding RNAs that act as negative regulators of gene expression [3]. miRNAs are abundant and ubiquitous, and impact almost all fundamental cell processes such as growth, differentiation, apoptosis, and adhesion [4]. Dysregulation of miRNA expression is a common feature in many types of human cancers, including thyroid cancer [5 6].

\section{RESULTS}

Study collection and inclusion

We initially collected 106 studies using the described strategy. 84 articles were excluded because they were not relevant to the aim of our study. 29 miRNAs in 16 articles were subsequently identified [7-23], with the function of dysregulated miRNAs confirmed through patient follow-up or other laboratory experiments. The dysregulated miRNAs are shown in detail in Tables 1a-1b. 


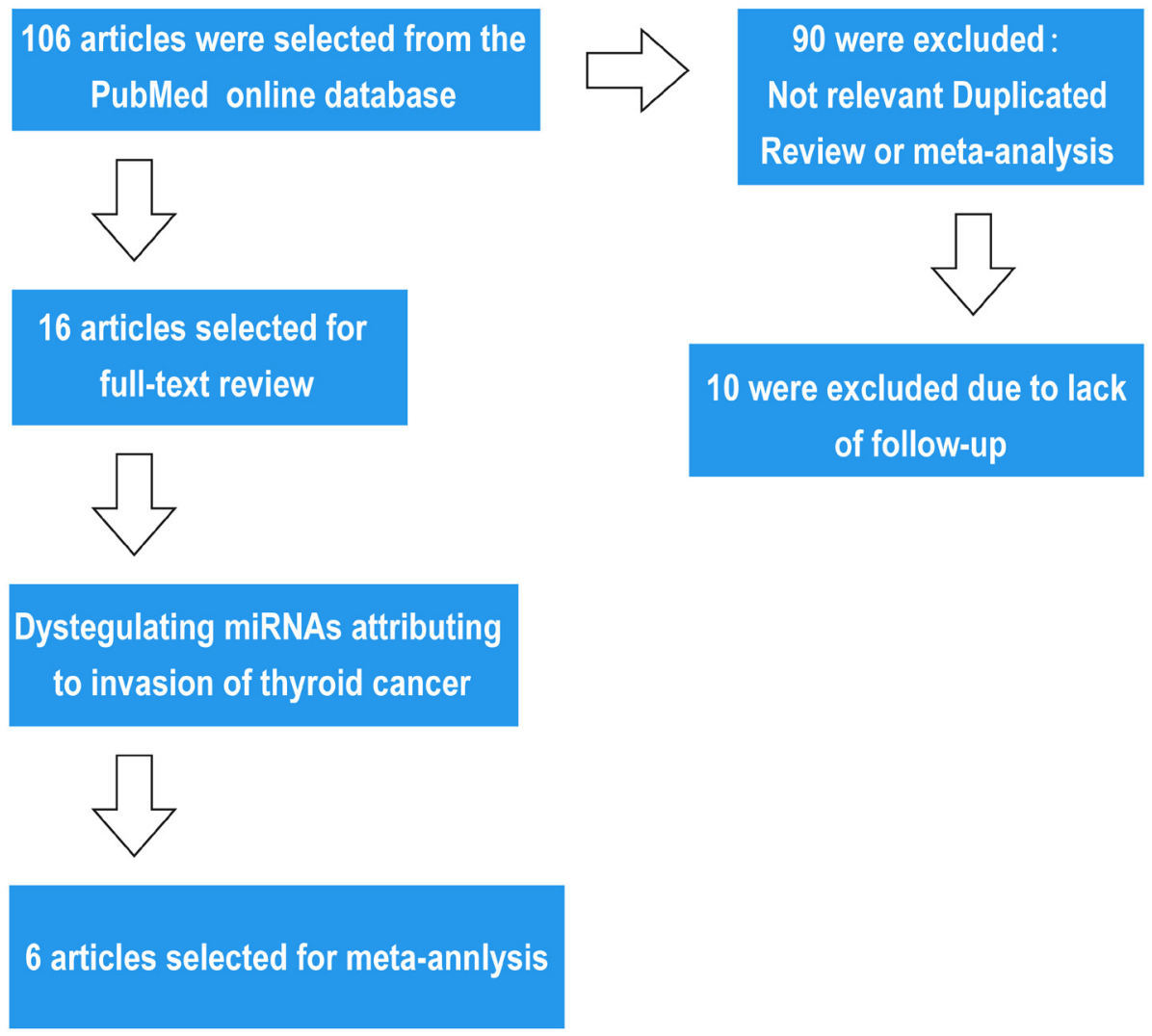

Figure 1: Flow chart of search strategy

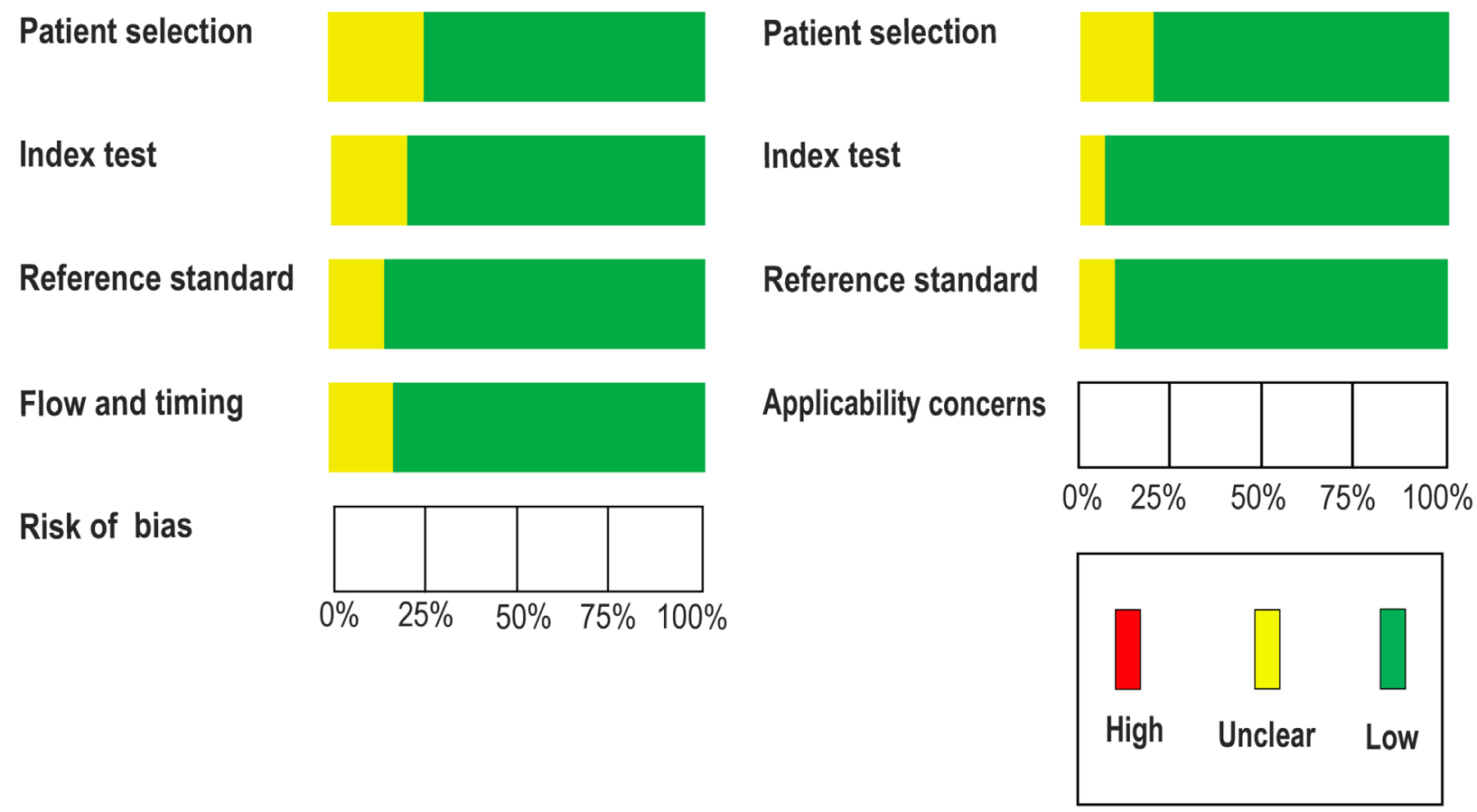

Figure 2: Quality of selected studies according to QUADAS-2 guidelines. 
Table 1a: Studies demonstrating an association between upregulated miRNAs and invasion

\begin{tabular}{|c|c|c|c|c|}
\hline miRNA & Author & Year & Country & Method \\
\hline \multirow{7}{*}{ MiRNA-146b } & Chou & 2010 & China & qRT-PCR \\
\hline & Chou & 2012 & China & qRT-PCR \\
\hline & Deng & 2015 & China & qRT-PCR \\
\hline & Lee & 2013 & Australia & Micro array \\
\hline & Lima & 2016 & Brazil & qRT-PCR \\
\hline & Yang & 2013 & China & Micro array \\
\hline & Wang & 2013 & China & Micro array \\
\hline \multirow{6}{*}{ MiRNA-222 } & Chou & 2010 & China & qRT-PCR \\
\hline & Lee & 2013 & Australia & Micro array \\
\hline & Jikuzono & 2013 & Africa & qRT-PCR \\
\hline & Yang & 2013 & China & Micro array \\
\hline & Chou & 2012 & China & qRT-PCR \\
\hline & Wang & 2013 & China & Micro array \\
\hline \multirow{6}{*}{ MiRNA-221 } & Chou & 2010 & China & qRT-PCR \\
\hline & Chou & 2012 & China & qRT-PCR \\
\hline & Jikuzono & 2013 & Africa & qRT-PCR \\
\hline & Wang & 2013 & China & Micro array \\
\hline & Zhou & 2012 & China & Northern blot \\
\hline & Yang & 2013 & China & Micro array \\
\hline MiRNA-4295 & Shao & 2015 & China & Micro array \\
\hline MiRNA-101 & Wang & 2014 & China & qRT-PCR \\
\hline MiRNA-183 & Wei & 2015 & China & qRT-PCR \\
\hline MiRNA-210 & Yang & 2013 & China & Micro array \\
\hline MiRNA-584 & Xiang & 2015 & China & qRT-PCR \\
\hline MiRNA-1244 & Yang & 2013 & China & Micro array \\
\hline MiRNA-134 & Yang & 2013 & China & Micro array \\
\hline MiRNA-214 & Yang & 2013 & China & Micro array \\
\hline MiRNA-1202 & Wang & 2013 & China & Micro array \\
\hline MiRNA-193 & Wang & 2013 & China & Micro array \\
\hline MiRNA-2861 & Wang & 2013 & China & Micro array \\
\hline
\end{tabular}

Table 1b: Studies demonstrating an association between downregulated miRNAs and invasion

\begin{tabular}{|l|c|c|c|c|}
\hline \multicolumn{1}{|c|}{ MiRNA } & Author & Year & Country & Method \\
\hline MiRNA-539 & GU & 2015 & China & qRT-PCR \\
\hline MiRNA-144 & Guan & 2014 & China & qRT-PCR \\
\cline { 2 - 5 } MiRNA-7 & Hua & 2016 & China & Micro array \\
\hline MiRNA-182 & Zhu & 2013 & China & Micro array \\
\hline MiRNA-126 & Xiong & 2014 & China & Micro array \\
\hline MiRNA-486 & Yang & 2013 & ChA & qRT-PCR \\
\hline MiRNA-206 & Zhang & 2015 & China & Micro array \\
\hline MiRNA-1302 & Yang & 2013 & China & Micro array \\
\hline MiRNA-1231 & Yang & 2013 & China & Micro array \\
\hline MiRNA-637 & Yang & 2013 & China & Micro array \\
\hline MiRNA-1826 & Yang & 2013 & China & Micro array \\
\hline MiRNA-1225 & Yang & 2013 & China & Micro array \\
\hline MiRNA-564 & Wang & 2013 & China & Micro array \\
\hline MiRNA-664 & Wang & 2013 & China & Micro array \\
\hline MiRNA-542 & Wang & 2013 & China & Micro array \\
\hline
\end{tabular}


Table 2: Studies included in the meta-analysis

\begin{tabular}{|l|c|l|l|l|c|}
\hline MiRNA & Author & Year & Country & Methods & Samples (tumor/control) \\
\hline \multirow{5}{*}{ MiRNA-146b } & Chou & 2010 & China & qRT-PCR & $16 / 16$ \\
\cline { 2 - 6 } & Chou & 2012 & China & qRT-PCR & $30 / 41$ \\
\cline { 2 - 6 } & Deng & 2015 & China & qRT-PCR & $30 / 30$ \\
\cline { 2 - 6 } & Lee & 2013 & Australia & Micro array & $9 / 17$ \\
\cline { 2 - 6 } & Yang & 2013 & China & Micro array & $3 / 3$ \\
\cline { 2 - 6 } & Wang & 2013 & China & Micro array & $3 / 3$ \\
\hline \multirow{4}{*}{ MiRNA-221 } & Chou & 2010 & China & qRT-PCR & $16 / 16$ \\
\cline { 2 - 6 } & Chou & 2012 & China & qRT-PCR & $30 / 41$ \\
\cline { 2 - 6 } & Lee & 2013 & Australia & Micro array & $9 / 17$ \\
\cline { 2 - 6 } & Yang & 2013 & China & Micro array & $3 / 3$ \\
\hline & Chou & 2010 & China & qRT-PCR & $16 / 16$ \\
\cline { 2 - 6 } & Chou & 2012 & China & qRT-PCR & $30 / 41$ \\
\cline { 2 - 6 } & Lee & 2013 & Australia & Micro array & $9 / 17$ \\
\cline { 2 - 6 } & Yang & 2013 & China & Micro array & $3 / 3$ \\
\hline
\end{tabular}

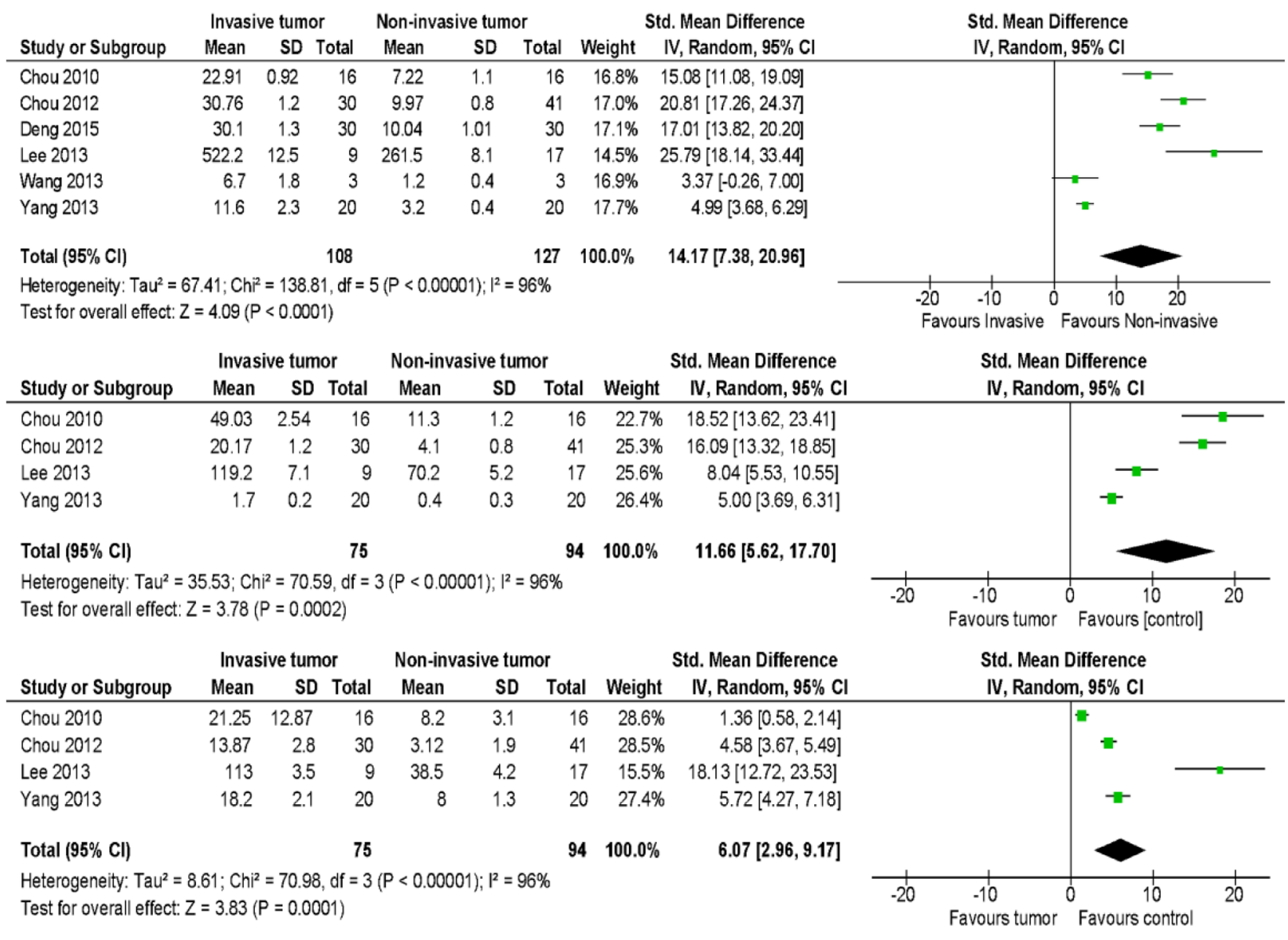

Figure 3: Forest plots for miRNA-146, miRNA-221, and miRNA-222 in the described articles. As $I^{2}>50 \%$, a randomeffects model was used. $p<0.05$, indicated that miRNA-146, miRNA-221, and miRNA-222 were all upregulated in invasive tumors compared with paired controls. 


\section{miRNA inclusion}

Among these miRNAs, miRNA-146b, miRNA-221, and miRNA-222 were analyzed further due to their appearance at higher frequency. Of the studies that analyzed these miRNAs, 6 were selected for meta-analysis because they compared invasive PTC with paired normal tissues or non-invasive PTC [7-12]. These dysregulated miRNAs are shown in detail in Table 2.

\section{Data characteristics}

The 6 included articles are shown in Table 2. In all, 201 samples were analyzed, including 91 invasive tumors and 110 paired control tissues (either non-invasive tumors or normal tissue).

\section{Meta-analysis results}

miRNA-146b was upregulated in all 6 studies, while miRNA-221 and miRNA-222 were upregulated in
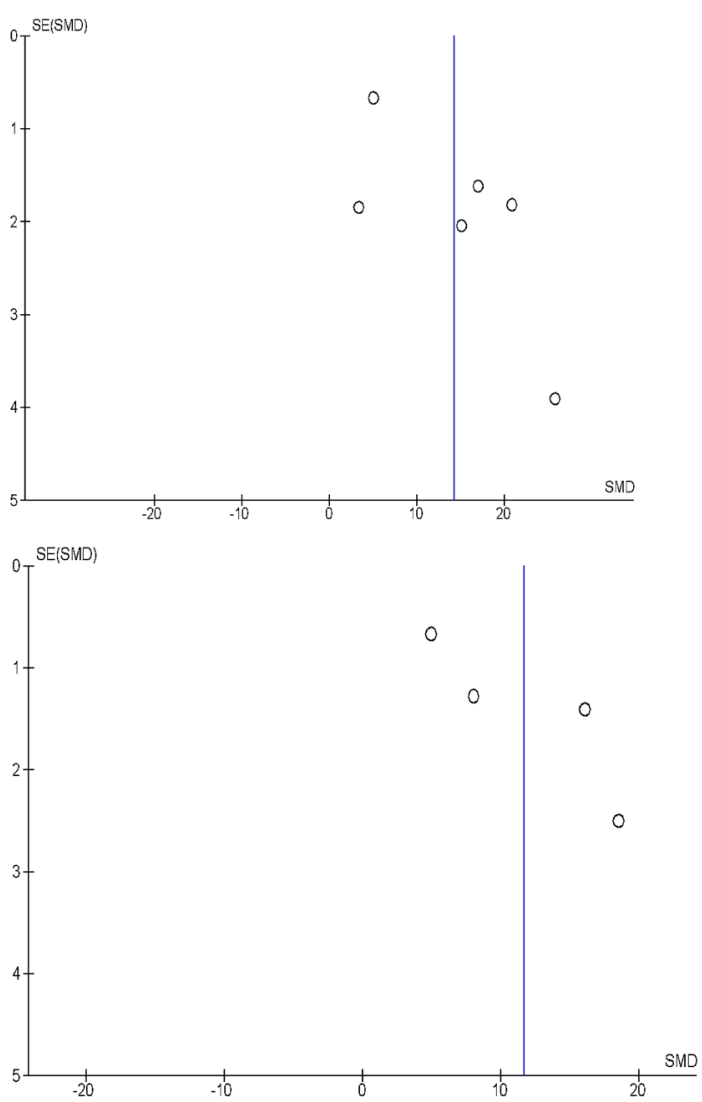

4 studies. The forest plot is shown in Figure 3. Given that $\mathrm{I}^{2}>50 \%$, the fixed-effects model was used. $p<0.00001$ for each miRNA was determined, suggesting that miRNA146b, miRNA-221, and miRNA 222 are all upregulated in invasive tumors compared with paired control.

\section{Publication bias}

Publication bias in our study was assessed using funnel plot analysis. As shown in Figure 4, there was minimal publication bias in this research, though some amount is unavoidable due to the lack of prior research.

\section{DISCUSSION}

Thyroid carcinoma (TC) is the most common endocrine malignancy. The overall survival rate of patients with thyroid carcinoma is high [1], however, increased cancer recurrence and cancer-related mortality are noted in a portion of patients with papillary thyroid carcinoma [2]. Distinguishing these tumors from classic non-lethal papillary tumors has therefore become a hot topic of

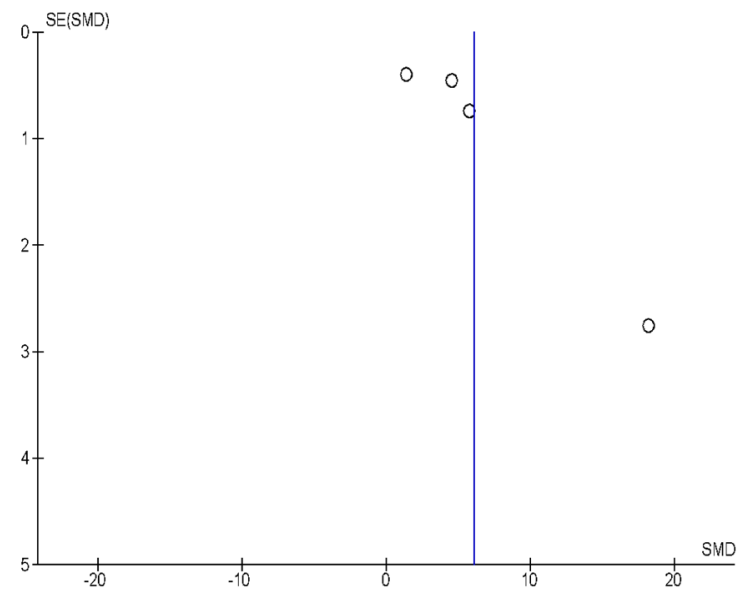

Figure 4: Funnel plot to measure publication bias, with regards to miRNA-146. Due to the relative lack of research studies, publication bias is somewhat unavoidable. 
research in recent years.

MicroRNAs (miRNAs) are a recently identified class of small, endogenous, non-coding RNAs that act as negative regulators of gene expression [3]. miRNAs are abundant and ubiquitous, and they impact almost all fundamental cell processes including growth, differentiation, apoptosis, and adhesion [4]. Dysregulation of miRNA expression is a common feature of many types of human cancers, including thyroid cancer [5 6].

It is well-known that many dysregulated miRNAs contribute to the tumorigenesis and progression of tumors. Many studies show a direct relationship between dysregulated miRNAs and the molecular mechanisms they induce to affect tumor invasion, but there has been less research addressing the clinical features surrounding invasion ability. In this article, we explored the relationship between dysregulated miRNAs and invasion ability. We searched the PubMed online database using the keywords "papillary thyroid carcinoma" or "thyroid carcinoma", "miRNA", and "invasion" and collected 106 records following the strategy described above. 84 articles were excluded because they were not relevant to the aim of our study. 29 miRNAs in 16 articles were identified with the function of the dysregulated miRNAs confirmed via patient follow-up or other laboratory experiments. Among these miRNAs, miRNA-146b, miRNA-221, and miRNA-222 were analyzed further. In total, 6 studies were included in the meta-analysis because they compared invasive PTC with paired normal tissues or non-invasive PTC.

The present meta-analysis has several limitations. Firstly, due to the lack of significant current research, only 6 articles comparing invasive PTC with paired noninvasive PTC or normal tissues were able to be included. Secondly, the divergence of incorporated studies also likely contributed to the statistical differences. Finally, there was a lack of strong correlation between overall patient survival statistics and the presence of dysregulated miRNAs due to lack of patient follow-up.

\section{MATERIALS AND METHODS}

\section{Search strategy}

A search for dysregulated miRNAs in papillary thyroid carcinoma was performed by querying the PubMed online database with the terms "miRNA" or "microRNA" or "miR", "thyroid carcinoma" or "papillary thyroid carcinoma", and "invasion". Titles and abstracts of the obtained articles were screened, and full texts of the articles of interest were further evaluated.

\section{Inclusion and exclusion criteria}

Research studies were considered to be eligible if they met the following criteria: (1) the study focused on patients with any type of papillary thyroid carcinoma; (2) the effect of the dysregulated miRNA on invasion was confirmed via analysis of the patients' metastases or other experimental tests in the same article; (3) for meta-analysis articles, miRNA effect was confirmed through patient follow-up. Articles were excluded based on the following criteria: (1) reviews, letters, comments, or pure laboratory studies; (2) absence of specific evidence showing the dysregulated miRNA had any influence on invasion; (3) discrepant conclusions across different articles.

\section{Statistical analysis}

The fixed-effects and random-effects models were used for the meta-analysis according to heterogeneity among the pooled studies. The heterogeneity test for the pooled odds ratio (OR) was verified using the $\mathrm{I}^{2}$ statistic. A random-effects model was applied if the heterogeneity was significant ( $p<0.01$ or $\mathrm{I}^{2}>50 \%$ ), while a fixed-effects model was applied if the heterogeneity was not significant $\left(p>0.01\right.$ or $\left.\mathrm{I}^{2}<50 \%\right)$. Publication bias was estimated using a funnel plot. All $p$ values were calculated using a two-sided test; differences were considered statistically significant when $p<0.05$. All statistical analyses were conducted using the Review Manager 5 (Cochrane Tech, London, UK) and Microsoft Excel (Microsoft Corporation, Seattle, WA, USA) software.

\section{CONCLUSIONS}

Current evidence and the findings of this article suggest, despite the limitations described above, that dysregulation of these miRNAs leads to increased invasion ability of thyroid carcinoma.

\section{CONFLICTS OF INTEREST}

The author declares no conflicts of interest.

\section{REFERENCES}

1. Torre LA, Bray F, Siegel RL, Ferlay J, Lortet-Tieulent J, Jemal A. Global cancer statistics, 2012. CA Cancer J Clin. 2015; 65: 87-108. doi: 10.3322/caac.21262.

2. Xing M, Alzahrani AS, Carson KA, Shong YK, Kim TY, Viola D, Elisei R, Bendlova B, Yip L, Mian C, Vianello F, Tuttle RM, Robenshtok E, et al. Association between BRAF V600E mutation and recurrence of papillary thyroid cancer. J Clin Oncol. 2015; 33: 42-50. doi: 10.1200/ JCO.2014.56.8253. 
3. Lagos-Quintana M, Rauhut R, Lendeckel W, Tuschl T. Identification of novel genes coding for small expressed RNAs. Science. 2001; 294: 853-8. doi: 10.1126/ science.1064921.

4. Mraz M, Malinova K, Mayer J, Pospisilova S. MicroRNA isolation and stability in stored RNA samples. Biochem Biophys Res Commun. 2009; 390: 1-4. doi: 10.1016/j. bbrc.2009.09.061.

5. Mitchell PS, Parkin RK, Kroh EM, Fritz BR, Wyman SK, Pogosova-Agadjanyan EL, Peterson A, Noteboom J, O'Briant KC, Allen A, Lin DW, Urban N, Drescher CW, et al. Circulating microRNAs as stable blood-based markers for cancer detection. Proc Natl Acad Sci U S A. 2008; 105: 10513-8. doi: 10.1073/pnas.0804549105.

6. Kim JO, Gazala S, Razzak R, Guo L, Ghosh S, Roa WH, Bedard EL. Non-small cell lung cancer detection using microRNA expression profiling of bronchoalveolar lavage fluid and sputum. Anticancer Res. 2015; 35: 1873-80.

7. Chou CK, Chen RF, Chou FF, Chang HW, Chen YJ, Lee YF, Yang KD, Cheng JT, Huang CC, Liu RT. miR-146b is highly expressed in adult papillary thyroid carcinomas with high risk features including extrathyroidal invasion and the BRAF(V600E) mutation. Thyroid. 2010; 20: 489-94. doi: 10.1089/thy.2009.0027.

8. Chou CK, Yang KD, Chou FF, Huang CC, Lan YW, Lee YF, Kang HY, Liu RT. Prognostic implications of miR$146 \mathrm{~b}$ expression and its functional role in papillary thyroid carcinoma. J Clin Endocrinol Metab. 2013; 98: E196-205. doi: 10.1210/jc.2012-2666.

9. Deng X, Wu B, Xiao K, Kang J, Xie J, Zhang X, Fan Y. MiR-146b-5p promotes metastasis and induces epithelialmesenchymal transition in thyroid cancer by targeting ZNRF3. Cell Physiol Biochem. 2015; 35: 71-82. doi: 10.1159/000369676.

10. Lee JC, Zhao JT, Clifton-Bligh RJ, Gill A, Gundara JS, Ip JC, Glover A, Sywak MS, Delbridge LW, Robinson BG, Sidhu SB. MicroRNA-222 and microRNA-146b are tissue and circulating biomarkers of recurrent papillary thyroid cancer. Cancer. 2013; 119: 4358-65. doi: 10.1002/ cncr.28254.

11. Yang Z, Yuan Z, Fan Y, Deng X, Zheng Q. Integrated analyses of microRNA and mRNA expression profiles in aggressive papillary thyroid carcinoma. Mol Med Rep. 2013; 8: 1353-8. doi: 10.3892/mmr.2013.1699.

12. Wang Z, Zhang H, He L, Dong W, Li J, Shan Z, Teng W. Association between the expression of four upregulated miRNAs and extrathyroidal invasion in papillary thyroid carcinoma. Onco Targets Ther. 2013; 6: 281-7. doi: 10.2147/OTT.S43014.

13. Lima CR, Geraldo MV, Fuziwara CS, Kimura ET, Santos MF. MiRNA-146b-5p upregulates migration and invasion of different Papillary Thyroid Carcinoma cells. BMC Cancer. 2016; 16: 108. doi: 10.1186/s12885-016-2146-z.

14. Jikuzono T, Kawamoto M, Yoshitake H, Kikuchi K, Akasu
H, Ishikawa H, Hirokawa M, Miyauchi A, Tsuchiya S, Shimizu K, Takizawa T. The miR-221/222 cluster, miR-10b and miR-92a are highly upregulated in metastatic minimally invasive follicular thyroid carcinoma. Int J Oncol. 2013; 42: 1858-68. doi: 10.3892/ijo.2013.1879.

15. Shao M, Geng Y, Lu P, Xi Y, Wei S, Wang L, Fan Q, Ma W. miR-4295 promotes cell proliferation and invasion in anaplastic thyroid carcinoma via CDKN1A. Biochem Biophys Res Commun. 2015; 464: 1309-13. doi: 10.1016/j. bbrc.2015.07.128.

16. Wang $\mathrm{C}$, Lu S, Jiang J, Jia X, Dong X, Bu P. HsamicroRNA-101 suppresses migration and invasion by targeting Rac1 in thyroid cancer cells. Oncol Lett. 2014; 8: 1815-21. doi: 10.3892/ol.2014.2361.

17. Wei C, Song H, Sun X, Li D, Song J, Hua K, Fang L. miR-183 regulates biological behavior in papillary thyroid carcinoma by targeting the programmed cell death 4 . Oncol Rep. 2015; 34: 211-20. doi: 10.3892/or.2015.3971.

18. Xiang J, Wu Y, Li DS, Wang ZY, Shen Q, Sun TQ, Guan Q, Wang YJ. miR-584 Suppresses Invasion and Cell Migration of Thyroid Carcinoma by Regulating the Target Oncogene ROCK1. Oncol Res Treat. 2015; 38: 436-40. doi: 10.1159/000438967.

19. Gu L, Sun W. MiR-539 inhibits thyroid cancer cell migration and invasion by directly targeting CARMA1. Biochem Biophys Res Commun. 2015; 464: 1128-33. doi: 10.1016/j.bbrc.2015.07.090.

20. Guan H, Liang W, Xie Z, Li H, Liu J, Liu L, Xiu L, Li Y. Down-regulation of miR-144 promotes thyroid cancer cell invasion by targeting ZEB1 and ZEB2. Endocrine. 2015; 48: 566-74. doi: 10.1007/s12020-014-0326-7.

21. Hua K, Jin J, Zhang H, Zhao B, Wu C, Xu H, Fang L. MicroRNA-7 inhibits proliferation, migration and invasion of thyroid papillary cancer cells via targeting CKS2. Int J Oncol. 2016; 49: 1531-40. doi: 10.3892/ijo.2016.3660.

22. Zhu H, Fang J, Zhang J, Zhao Z, Liu L, Wang J, Xi Q, Gu M. miR-182 targets CHL1 and controls tumor growth and invasion in papillary thyroid carcinoma. Biochem Biophys Res Commun. 2014; 450: 857-62. doi: 10.1016/j. bbrc.2014.06.073.

23. Xiong Y, Kotian S, Zeiger MA, Zhang L, Kebebew E. miR-126-3p Inhibits Thyroid Cancer Cell Growth and Metastasis, and Is Associated with Aggressive Thyroid Cancer. PLoS One. 2015; 10: e0130496. doi: 10.1371/ journal.pone.0130496. 